\title{
ERRATUM
}

\section{Erratum to: Special primitive pairs in finite groups}

\author{
REBECCA WALDECKER
}

\begin{abstract}
During the review of the article "Special primitive pairs in finite groups", an error was found in the proof of one of the preliminary lemmas. This note corrects this error and the author wishes to thank George Glauberman for pointing it out.
\end{abstract}

Mathematics Subject Classification. Primary 20Fxx; Secondary 20Exx.

Keywords. Finite groups, Primitive pairs, Characteristic $q$.

Erratum to: Arch. Math. 97 (2011), 11-16

DOI 10.1007/s00013-011-0271-0

Throughout this note let $X$ be a group, let $\pi$ be a set of primes, and let $r$ be a prime number. We change Lemmas 2.3 and 2.4 in the article slightly and give an explicit proof of the new Lemma 2.3. Then the hypothesis of Lemma 2.4 and its proof are adapted. The changes are minor, and the rest of the paper is unaffected because in all appeals to Lemma 2.4, its new hypothesis that $A \leq Z_{\pi}^{*}(X)$ is automatically satisfied. In the proofs of Lemmas 2.7 and 3.2, the comments that $A \leq O_{\pi^{\prime}, \pi}(H)$ (or $A \leq O_{\pi^{\prime}, \pi}(W)$ ) should therefore be ignored.

Lemma 2.3. (new) Suppose that $A$ is a $\pi$-subgroup of $Z_{\pi}^{*}(X)$. Then $O_{\pi^{\prime}}\left(C_{X}(A)\right)$ $\leq O_{\pi^{\prime}}(X)$.

Proof. As $A \leq Z_{\pi}^{*}(X)$, we have that $X=C_{X}(A) O_{\pi^{\prime}}(X)$. Let $\bar{X}:=X / O_{\pi^{\prime}}(X)$. Then $\bar{A} \leq Z(\bar{X})$ and therefore $\bar{X}=C_{\bar{X}}(\bar{A})=\overline{C_{X}(A)}$. We conclude that

The online version of the original article can be found under doi:10.1007/s00013-011-0271-0. 


$$
O_{\pi^{\prime}}\left(\overline{C_{X}(A)}\right)=O_{\pi^{\prime}}(\bar{X})=1,
$$

and hence $O_{\pi^{\prime}}\left(C_{X}(A)\right) \leq O_{\pi^{\prime}}(X)$ as stated.

Lemma 2.4. (new) Suppose that $r \notin \pi$ and that $A$ is a $\pi$-subgroup of $Z_{\pi}^{*}(X)$. Then $X$ has a unique maximal $A C_{X}(A)$-invariant $r$-subgroup $R$ and $O_{r}(X) O_{r}\left(C_{X}(A)\right) \leq R \leq O_{\pi^{\prime}}(X)$.

Proof. Let $Y \in \mathrm{И}_{X}\left(A C_{X}(A), r\right)$ be arbitrary. The coprime action of $A$ on $Y$ yields that $Y=C_{Y}(A)[Y, A]$, with Lemma 2.1(1). As $C_{Y}(A)$ is a $C_{X}(A)$-invariant $r$-subgroup of $C_{X}(A)$ and $r \notin \pi$, Lemma 2.3 gives that

$$
C_{Y}(A) \leq O_{\pi^{\prime}}\left(C_{X}(A)\right) \leq O_{\pi^{\prime}}(X) .
$$

We also see that $[Y, A] \leq Y \cap Z_{\pi}^{*}(X) \leq O_{\pi^{\prime}}(X)$ because $A$ is a subgroup of $Z_{\pi}^{*}(X)$ and $Y$ is a $\pi^{\prime}$-group. Therefore every member of $\eta_{X}\left(A C_{X}(A), r\right)$ lies in $O_{\pi^{\prime}}(X)$. Together with the coprime action of $A$ on $O_{\pi^{\prime}}(X)$ and Lemma 2.2 this implies that the intersection $R$ of all $A$-invariant Sylow $r$-subgroups of $O_{\pi^{\prime}}(X)$ is the unique maximal $A C_{X}(A)$-invariant $r$-subgroup of $X$. As $O_{r}(X) O_{r}\left(C_{X}(A)\right)$ is, of course, an $A C_{X}(A)$-invariant $r$-subgroup of $X$, it is contained in $R$ as stated.

\section{REBECCA WALDECKER}

Institut für Mathematik,

Martin-Luther-Universität Halle-Wittenberg,

Theodor-Lieser-Straße 5,

06120 Halle (Saale),

Germany

e-mail: rebecca.waldecker@mathematik. uni-halle.de

Received: 13 February 2012 